\title{
Coronary Subclavian Steal Syndrome With Neurological Symptoms After Coronary Artery Bypass Grafting
}

\author{
Megan C. Smith ${ }^{1}$, Rich Pham ${ }^{1}$, Nicholas Coffey ${ }^{2}$, Mohammed Kazimuddin ${ }^{1}$, Aniruddha Singh ${ }^{1}$ \\ 1. Cardiology, The Medical Center/University of Kentucky, Bowling Green, USA 2. Cardiology, University of Kentucky \\ College of Medicine, Bowling Green, USA
}

Corresponding author: Megan C. Smith, megtail04@gmail.com

\begin{abstract}
Coronary subclavian steal syndrome (CSSS) is a complication of coronary artery bypass graft surgery with the left internal mammary artery that results from left subclavian artery stenosis. A reversal of flow in the left internal mammary artery results in ischemia of the heart. We present the case of a 54-year-old man with CSSS with the rare symptom of dizziness. This indicates a potential component of undiagnosed vertebral steal syndrome as well.
\end{abstract}

Categories: Cardiac/Thoracic/Vascular Surgery, Cardiology

Keywords: coronary subclavian steal syndrome, vertebral steal syndrome, coronary artery bypass graft

\section{Introduction}

Coronary subclavian steal syndrome (CSSS) is a complication that occurs after coronary artery bypass graft (CABG) surgery with the left internal mammary artery (LIMA). This complication arises from a reversal of flow in the grafted LIMA as a result of proximal subclavian artery stenosis or occlusion that "steals" blood from the heart. This pathological mechanism generally results in angina and upper extremity claudication $[1,2]$. Even with this complication, grafting the LIMA to the left anterior descending artery has become the most common graft in CABG. This is because it has superior survivability and durability compared to a saphenous vein graft (SVG).

Review began $12 / 25 / 2020$ Review ended 01/18/2021 Published 01/21/2021

๑) Copyright 2021

Smith et al. This is an open access article distributed under the terms of the Creative Commons Attribution License CC-BY 4.0., which permits unrestricted use, distribution, and reproduction in any medium, provided the original author and source are credited.

\section{Case Presentation}

A 54-year-old Caucasian male presented to the emergency department with severe chest pain, dyspnea, and dizziness along with left arm pain and weakness. The symptoms occurred while he attempted to install an overhead light fixture. His perceived dizziness was further described as lightheadedness and presyncopal symptoms, which always occurred when he was working with his left arm, particularly if his left arm was raised above his head. He stated that his left arm pain and weakness had been progressive to the point that he could no longer work as he could not hold his tools. Previously, he had been seen in the outpatient cardiology clinic multiple times throughout the last two years with complaints of recurrent angina. However, despite medical recommendations, he never underwent a coronary angiogram or stress test for further evaluation due to severe fear and anxiety associated with these procedures.

Past medical history was significant for hypertension, diabetes, hyperlipidemia, smoking, anxiety, and ischemic cardiomyopathy. A component of previous treatment for the ischemic cardiomyopathy was a fourvessel CABG in 2008, achieved by grafting the LIMA to the left anterior descending artery and SVGs to a diagonal branch, obtuse marginal branch, and posterior descending artery. Vitals were significant for a 23 $\mathrm{mmHg}$ difference in systolic blood pressures obtained from bilateral upper extremities, and physical examination revealed diminished pulses in the left arm, raising the suspicion for subclavian stenosis. Coronary and bypass angiography was performed due to concerns of unstable angina and revealed $100 \%$ occlusion of the proximal left subclavian artery (Figure 1). 


\section{Cureus}

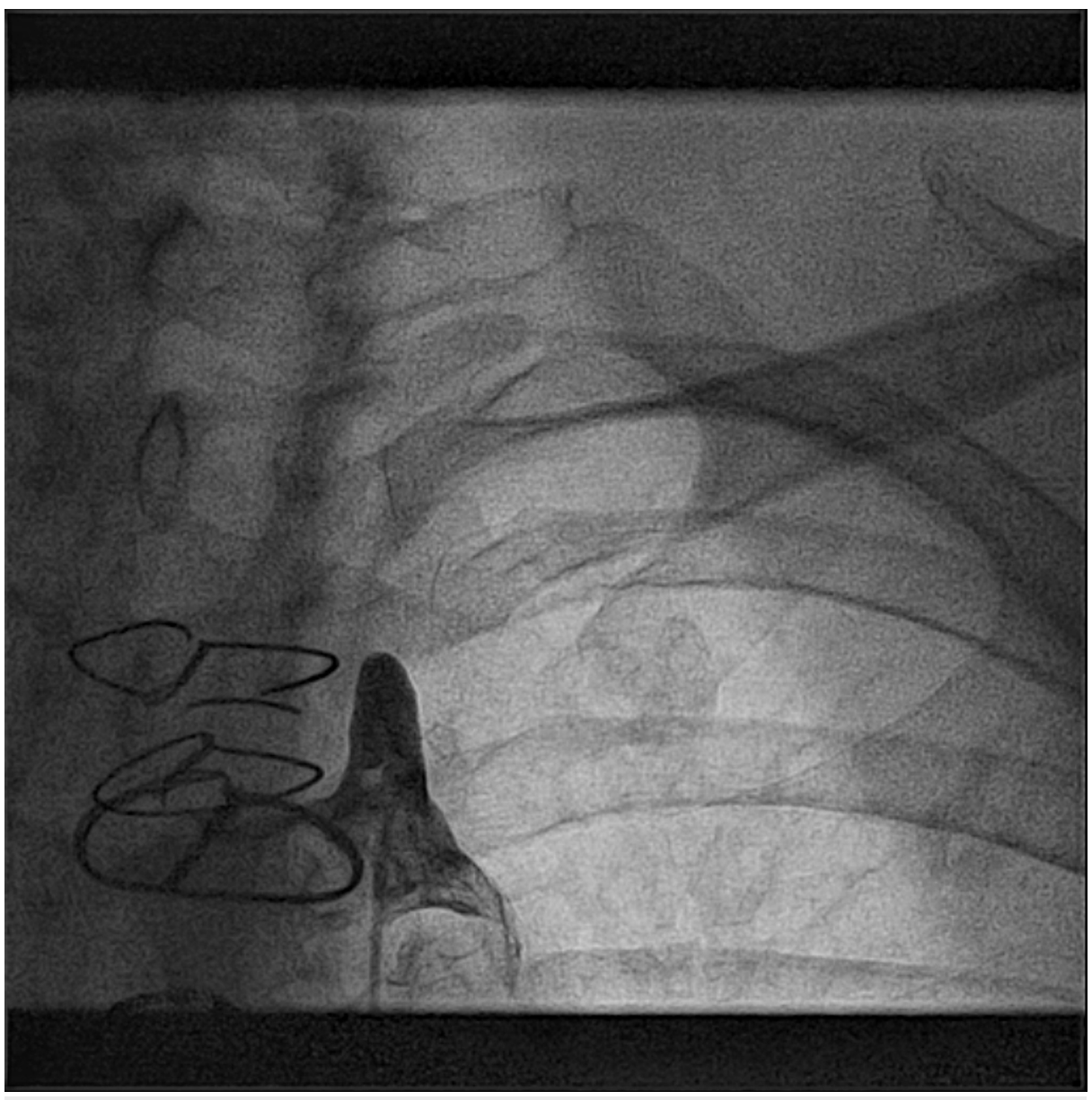

FIGURE 1: Angiogram of the proximal left subclavian artery occlusion.

Angiogram confirmed complete flow reversal from the proximal left subclavian artery to the LIMA (Video 1 ).

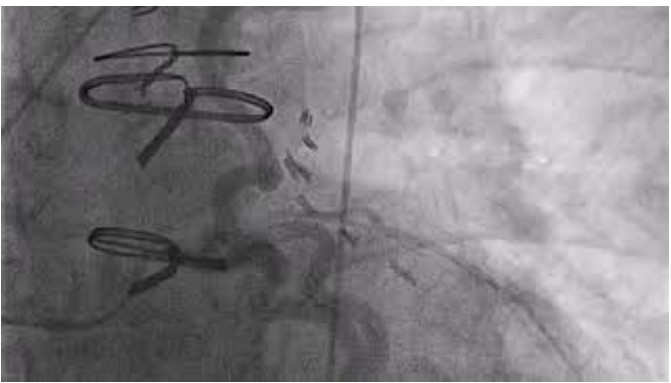

\section{VIDEO 1: Angiogram demonstrating complete flow reversal from the proximal left subclavian artery to the LIMA.}

LIMA: left internal mammary artery

View video here: https://youtu.be/zVvRr0j6tJ0

The LIMA had dilated from approximately its average size of $2 \mathrm{~mm}$ to $10 \mathrm{~mm}$, which is approximately the average size of the subclavian artery. Bilateral carotid duplex revealed no hemodynamically significant stenosis. Left carotid-subclavian bypass surgery was performed which relieved his exertional angina along with dizziness and left arm discomfort.

\section{Discussion}

CSSS was first described in 1974 and is a relatively rare disease with a prevalence of $2.5 \%$, as defined by at least 50\% left subclavian stenosis and a greater than $20 \mathrm{mmHg}$ brachial pressure difference in those that 
have undergone LIMA CABG [3,4]. However, all patients who presented in the study with CSSS were asymptomatic [4]. Our patient presented with classic symptoms of CSSS with unstable angina (present in $30.1 \%$ of the cases) and upper extremity claudication (present in $43.3 \%$ of the cases). However, dizziness is a rare symptom that may indicate a component of vertebral steal syndrome that results in neurological symptoms. Vertebrobasilar insufficiency can manifest as syncope, dizziness, vision changes, or tinnitus [1]. Transient ischemic attacks and cerebrovascular events occur infrequently, with hemispheric events occurring most frequently in patients with concomitant carotid lesions [4]. Other case reports with neurologic symptoms describe patients presenting with isolated dizziness, recurrent vertigo, and hearing changes [5].

The key diagnostic testing for subclavian steal syndrome, which is the overarching disease that CSSS falls under, is a difference in brachial artery blood pressure of $20 \mathrm{mmHg}$, with $78-88 \%$ of those with subclavian steal syndrome having this presentation [6]. Those with higher levels of pressure difference generally have worse symptoms [7]. Imaging is used to make a definitive diagnosis through such modalities as ultrasound, magnetic radiographic imaging angiogram, and computed tomography angiography [8].

Intervention is usually performed because of potential ischemia. Intervention generally consists of either carotid-subclavian bypass surgery or endovascular therapy, such as stent placement [1]. Carotid-subclavian bypass has been shown to have operational success rates of $98.11 \%$ with $100 \%$ graft patency rate approximately two years later $[9,10]$. Similarly, endovascular therapy, such as stents, were reported to have $93 \%$ patency after three years for subclavian stenosis cases $[9,11]$. However, carotid-subclavian bypass surgery is still considered the gold standard as a direct comparison study found that stent-supported percutaneous transluminal angioplasty, which is a form of endovascular therapy, failed in $48 \%$ of occlusions, while all carotid-subclavian bypass surgeries succeeded in approximately a 50-month follow-up on average $[9,12]$.

\section{Conclusions}

Symptomatic CSSS is a rare complication associated with LIMA CABG that results in the "stealing" of blood by the subclavian artery. This usually presents with upper arm claudication and unstable angina as described in this case. However, this case describes an even rarer entity where CSSS was associated with neurological symptoms that could be a result of vertebral steal syndrome, which has only been reported in a few case reports in the literature.

\section{Additional Information \\ Disclosures}

Human subjects: Consent was obtained or waived by all participants in this study. Conflicts of interest: In compliance with the ICMJE uniform disclosure form, all authors declare the following: Payment/services info: All authors have declared that no financial support was received from any organization for the submitted work. Financial relationships: All authors have declared that they have no financial relationships at present or within the previous three years with any organizations that might have an interest in the submitted work. Other relationships: All authors have declared that there are no other relationships or activities that could appear to have influenced the submitted work.

\section{References}

1. Sintek M, Coverstone E, Singh J: Coronary subclavian steal syndrome. Curr Opinion Cardiol. 2014, 29:506513. 10.1097/HCO.0000000000000109

2. Cua B, Mamdani N, Halpin D, Jhamnani S, Jayasuriya S, Mena-Hurtado C: Review of coronary subclavian steal syndrome. J Cardiol. 2017, 70:432-437. 10.1016/j.jjcc.2017.02.012

3. Harjola PT, Valle, M: The importance of aortic arch or subclavian angiography before coronary reconstruction. Chest. 1974, 66:436-438. 10.1378/chest.66.4.436

4. Hwang HY, Kim JH, Lee W, Park JH, Kim KB: Left subclavian artery stenosis in coronary artery bypass: prevalence and revascularization strategies. Ann Thorac Surg. 2010, 89:1146-1150. 10.1016/j.athoracsur.2010.01.007

5. Psillas G, Kekes G, Constantinidis J, Triaridis S, Vital V: Subclavian steal syndrome: neurotological manifestations. Acta Otorhinolaryngol Ital. 2007, 27:33-37.

6. Hennerici M, Klemm C, Rautenberg W: The subclavian steal phenomenon: a common vascular disorder with rare neurologic deficits. Neurology. 1988, 38:669-673. 10.1212/wnl.38.5.669

7. Labropoulos N, Nandivada P, Bekelis K: Prevalence and impact of the subclavian steal syndrome. Ann Surg. 2010, 252:166-170. 10.1097/SLA.0b013e3181e3375a

8. Potter BJ, Pinto DS: Subclavian steal syndrome. Circulation. 2014, 129:2320-2323. 10.1161/CIRCULATIONAHA.113.006653

9. Osiro S, Zurada A, Gielecki J, Shoja MM, Tubbs RS, Loukas M: A review of subclavian steal syndrome with clinical correlation. Med Sci Monit. 2012, 18:57-63. 10.12659/msm.882721

10. Qi L, Gu Y, Zhang J, et al.: Surgical treatment of subclavian artery occlusion [Article in Chinese] . Zhongguo Xiu Fu Chong Jian Wai Ke Za Zhi. 2010, 24:1030-1032.

11. De Vries JPP, Jager LC, Van den Berg JC, Overtoom TTC, Ackerstaff RGA, Van de Pavoordt EDWM, Moll FL: Durability of percutaneous transluminal angioplasty for obstructive lesions of proximal subclavian artery: 


\section{Cureus}

long-term results. J Vasc Surg. 2005, 41:19-23. 10.1016/j.jvs.2004.09.030

12. Linni K, Ugurluoglu A, Mader N, Hitzl W, Magometschnigg H, Hölzenbein TJ: Endovascular management versus surgery for proximal subclavian artery lesions. Ann Vasc Surg. 2008, 22:769-775.

10.1016/j.avsg.2008.08.001 